\title{
Cultural variations in virtual spaces design
}

Antonella De Angeli

\author{
Manchester Business School - University of Manchester \\ Booth Street west, Manchester, UK - M15 6PB, UK \\ 0044 (0)1613063383 - antonella.de-angeli@manchester.ac.uk
}

\begin{abstract}
This paper reports two studies investigating the role of culture on the design and personalisation of virtual spaces. The first study was a systematic analysis of $60 \mathrm{MSN}$ virtual spaces belonging to British and Chinese students. The analysis concentrated on design patterns and communication style. The second study was an on-line survey designed to compare the relative importance of cultural values and personality traits on self-reported behaviour with, and preferences for, virtual space design. Results highlighted the importance of culture on design and communication in virtual spaces. Implications for interaction design are discussed.
\end{abstract}

\section{Introduction}

The study of cultural differences and their impact on people's behaviour is a relatively new field of research in psychology (Markus and Kitayama 1991; Triandis 1989) and is still in its infancy in human-computer interaction (HCI). There is a growing consensus, however, that the user's cultural background is an important factor to be considered when designing and evaluating interactive devices (De Angeli et al. 2004; De Angeli and Kyriakoullis 2006; Efendioglu and Yip 2004; Marcus 2002; Smith and Yetim 2004). Yet, the precise direction and implications of this effect are still to be understood and translated into practical guidelines.

According to (Barber and Badre 1998), a systematic analysis of international websites may inform the identification of culturally-specific design standards and preferences. Following this proposal, this paper presents the analysis of cultural markers in the design of personal pages in social networking platforms (SNP's). SNP's, such as MSN spaces or Facebook (Lampe et al. 2007), offer standardised design environments used by end-users to build personal pages. SNP's are an example of socially-motivated personalization (Blom and Monk 2003) which provides a vast repository of aesthetical preferences and design stereotypes of a culturally diverse population. As compared to the web in general, SNP's simplify the identification of cultural markers by constraining the variability of genre and providing explicit information on the authors' background ${ }^{1}$. A further advantage relates to the specific nature of the content disclosed. Virtual spaces are designed to broadcast the author's identity in the cyberspace. Hence, it is reasonable to expect that they will mirror self-presentation processes of face-to-face encounters, and the analysis can be grounded in a robust theoretical background (Goffman 1959; Leary 1996).

\footnotetext{
${ }^{1}$ Deception cannot be excluded but it is believed to be a phenomenon of marginal relevance in social networks, especially when they have real-life connections or are restricted by invitation (Lampe et al 2007).
} 
This paper addresses the effect of cultural values and personal traits on virtual spaces design. Section 2 reports related works including (a) a summary of HCI research on cultural variations in design preferences; (b) major frameworks proposed in the social sciences to account for cultural differences in selfperception and communication style; and; (c) a review of self-presentation processes in real life and in computer-mediated communication. This theoretical background is used to inform two opposite hypotheses on the persistence of cultural variations in virtual spaces which are tested in two studies. The first study was a systematic analysis of $60 \mathrm{MSN}$ spaces belonging to British or Chinese students. The evaluation concentrated on design patterns and communication features. The second study was an on-line survey collecting self-reports about virtual spaces personalisation and preferences for different design styles. This study investigated the relative importance of cultural values and personality traits on design. The final section concludes and provides suggestion for future research.

\section{Theoretical background}

Culture is the common deposit of knowledge, meanings, beliefs, and values that unify a social group and differentiate it from others (Hofstede 2001). It is a forma mentis, a lens which mediates perception and action by providing conventions to sample information in the environment (Nisbett 2003). The interest in culturalaware design was originally motivated by the need to export products, designed for western markets, into different countries (De Angeli et al. 2004). A more recent movement unfolds the attention to cultural diversity in the concept of design values (Dearden et al. 2007). This view proposes to use HCI knowledge to foster international development by building devices which could be used by a diverse population, independently of literacy skills, previous knowledge, and environmental conditions.

A growing corpus of research has demonstrated that design features that are appropriate for one culture may not be appropriate for another, as cultural preferences and biases influence the user experience (De Angeli et al. 2004; Efendioglu and Yip 2004; Smith and Yetim 2004). Writing protocols, for instance, affect menu design (Dong and Salvendy 1999). Cultural values and habits shape meanings and values given to technological devices as simple as cash-machines (De Angeli et al. 2004). Different user requirements and needs were found in the context of e-commerce, highlighting a preference friend recommendations in collectivistic countries than in individualistic ones (De Angeli and Kyriakoullis 2006).

\subsection{Differences in self-construal}

Cultural differences in communication and behaviour are well documented in the psychological literature (Banaji 1994; Markus and Kitayama 1991; Triandis 1989). Recent advances in neuroimaging techniques have strengthened the hypothesis that cultural differences influence also lower level cognitive tasks such as perception. Differences were evidenced in direct neural activity during perception tasks, particularly for focal objects at an early stage of stimuli encoding (Gutchess et al. 2006). The brain parts activated by the same visual stimulation differed according to the perceiver's cultural background. 
An important psychological framework relates cultural differences in cognition, emotion and motivation to different form of self-construal. The self is a psychological construct used to denote all the knowledge people have about their self (Banaji 1994; Turner 1987). This knowledge represents the most important set of cognitive representations available to an individual, as the self directly acts as an information processor which mediates perception of the world. Knowledge about the self filters, interprets, and evaluates all the incoming stimuli in terms of their contribution to the individual's well being. As such, the self-concept is an influential determinant of cognition and action.

People in different cultures have different conceptions of the self. These representations are modulated on a continuum according to the interdependence between the information describing the self and that describing others (Markus and Kitayama 1991). In Eastern cultures (e.g., Asia) people tend to have a more inter-dependent self, whereas in western cultures (e.g., Europe and the United States) people tend to have a more independent self. The inter-dependent self is grounded in one's connection with others. Information about other people is stored as central nodes in the self-concept, and people tend to identify themselves through their roles and relationships (e.g., Son of X; Mother of Y). Main behavioural drivers are to fit in and to belong. The inter-dependent self is flexible and can easily adapt to the social context. People are concerned about others and favour others' goals to their own in order to maintain harmony.

The independent self is the opposite. It is grounded in autonomy, stability and uniqueness. Other people are still important but mainly for social comparison as they are external to the self. The independent self is expressed through one's inner thoughts and feelings. People with an independent self use a direct communication style as they are driven by the realisation of personal goals and the manifestation of individual capabilities.

The theoretical distinction between independent and inter-dependent self, based on the relative importance given to individual versus relational connections, is explicit in several other cultural frameworks, including Hofstede's cultural value dimension of collectivism versus individualism (Hofstede 2001).

\subsection{Differences in communication style}

A number of theoretical perspectives suggest that, transcending linguistic differences, communication is strongly influenced by the speaker's cultural background. An influential paradigm is represented by the distinction between low-context and high-context communication culture (Hall 1976). Context refers to the background knowledge a person needs to have in order to communicate effectively. Cultures vary according to the extent to which a message is explicitly conveyed by language.

In high-context societies, such as most Eastern countries, greater importance is placed in non-verbal aspects of communication, (such as body language, vocal tone, and the use of silence) than in verbal aspects. High-context cultures emphasizes listening and interpretation over direct explanation. Understanding relies heavily on the receiver's ability to decipher the message using contextual cues to fill in gaps. Low-context cultures, on the other hand, use an explicit communication code and place emphasis on the ability of the speaker to use direct 
verbal cues for effective communication. The main differences between lowcontext and high-context can be summarised on the continuum implicit -explicit, reserved - open, and ambiguous - clear.

\subsection{Self-presentation processes}

Self-presentation and self-disclosure processes are fundamental aspects of social behaviour. The difference between the two terms relates to the objective of the communication act (Johnson 1981). Self-presentation refers to information given to an audience on how the speaker has to be regarded. Self-disclosure refers to factual information about the self given by the speaker to an audience. The main difference regards the possibility of deception (more likely in self-presentation) and intimacy (more strongly associated to self-disclosure). In modern psychological discourse, self-presentation is regarded as a broader concept encompassing and enlarging that of self-disclosure. For this reason, we favour the term self-presentation in this paper.

In face-to-face encounters, selves are construed, modified and enacted in relation to an audience. Goffman's seminal work on face-to-face interaction links this process to theatre, stating that people play different roles for different audiences (Goffman 1959). The actor aims to convey an impression on the audience which is in their best interest to convey (Leary 1996). In Goffman's words, they intentionally give out information to maintain a positive self-image. This information is under the intentional control of the speaker, such as the verbal channel. However, unintentional information is often given off by speakers. It leaks through the communication in the form of non verbal behaviour, such as speech tone, facial expressions, and dressing styles (Goffman 1959).

Important individual and cultural differences in self-presentation are well documented in real-life (Banaji 1994; Leary 1996; Markus and Kitayama 1991; Triandis 1989) and more recently in computer-mediated communication (Marcus et al. 2006; Wood and Smith 2005). Social identity theorists (Tajfel and Turner 1986; Turner 1987) propose a differentiation between two broad classes of self, namely personal identity (the self as an individual) and social identity (the self as a group member). Activation of self-representation, or self-categorisation, is context dependent. Emphasis on personal identity leads people to behave as unique individuals; emphasis on social identity leads people to behave as group members. The idea that we have different selves, and that these selves can be elicited by contextual factors, has several important implications for selfpresentation, as it defines the set of information available for disclosure (Banaji 1994). This is especially important in on-line contexts, where presenters may face uncertain audience from the whole virtual world, not anticipated subjects. In this context, the regulatory function of the audience is lost or, anyway, substantially delayed.

The lack of direct social cues regulating self-presentation on-line has lead to a conceptualisation of the Internet as a medium with the power of liberating individuals from social boundaries and influences, reducing cultural and power differences, and allowing a more egalitarian exchange of information than face-toface encounters (Sproull and Kiesler 1992). In this view, it is believed that the Internet emphasises the individual identity, stressing people's unique personality 
and decreasing the importance of other social factors, such as culture. Following this line of thinking, we can postulate the following hypothesis.

Hypothesis 1-a. Cultural differences will be mitigated in virtual spaces.

An opposite line of research stressed the social nature of computer-mediated communication, claiming that the lack of subtle cues, allowing differentiation between members of a group in face-to-face encounters, may foster social categorisation and stereotyping based on easily available characteristics of the communicator, such as gender, ethnicity and nationality (Lea 1991; Postmes et al. 1998). This viewpoint states that, whenever the author's social identity is salient, on-line communication is ruled by social norms. Virtual spaces immediately disclose social information about the author, visually (e.g., pictures) and verbally (e.g., profile), whereas communication and perception of idiosyncratic characteristics of individuals may require more effort. Hence, virtual spaces can accentuate the perception of the author's social identity leading them to follow more closely expected rules of conduct. This line of thinking leads to the proposition of an opposite hypothesis.

Hypothesis 1-b. Cultural differences in communication style will be strengthened in virtual space.

\section{Virtual spaces analysis}

This paper focuses on MSN spaces, a SNP launched in 2004 by Microsoft Corporation $^{2}$. This choice was due to the incredible success of the platform at the time of the study (spring 2006) and to its personalization features. Using virtual spaces users could present their self through direct communication (e.g., the text in their blog or the pictures in their photo album) and through indirect communication (e.g., interface design). This difference is important in selfpresentation as it resembles Goffman's distinction between information given and information given off. Examples of virtual spaces are reported in Figure 1.
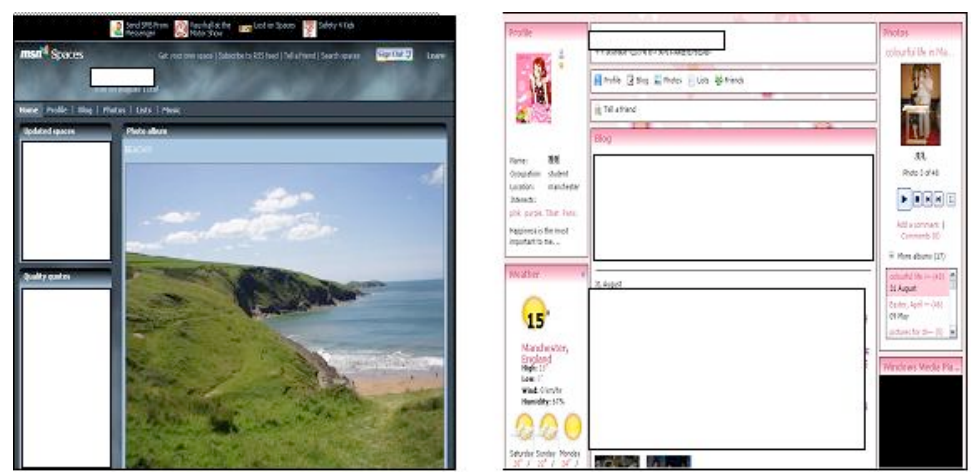

Fig. 1. MSN spaces examples (text is deleted to protect privacy)

At the time of the study, MSN spaces provided a set of modules to populate a personalized multimedia environment. These modules included profile, blog, photo album, window media player, music list, custom list, and book list. Each module was displayed in a self-contained window (Figure 2), which the user could position in the screen. MSN spaces offered six pre-set graphical layouts,

2 In August 2006 MSN Spaces were substituted by Windows Live Spaces 
some hundreds graphical themes, and a broad choice of colours. Users were given the option to set access rights for their space.
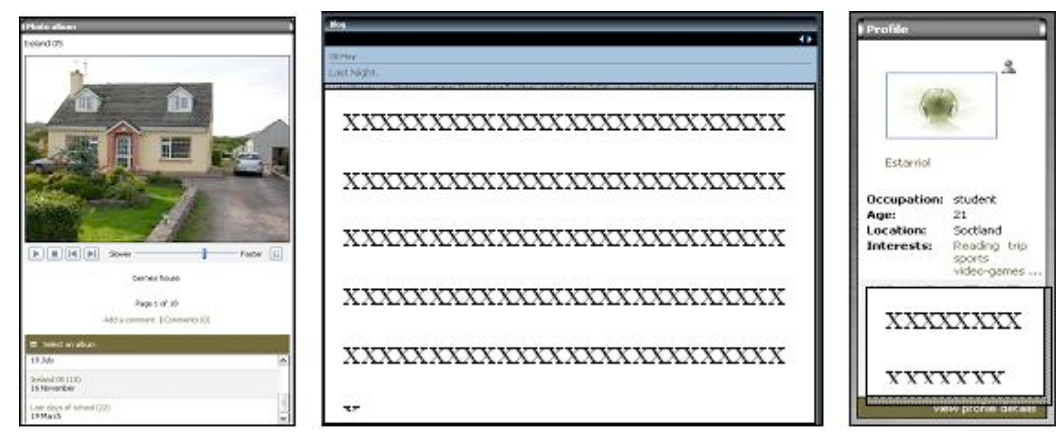

Fig. 2. Examples of photo album, blog, and profile module (text is deleted to protect privacy)

\subsection{Method}

The study consisted of a systematic analysis of real spaces addressing design patterns and communication features. Spaces were selected to satisfy the following requirements. The authors had to be students of the University of Manchester, who were born and raised in the UK or in China (the average Chinese participants had spent 1.5 year in the UK). This constraint was introduced to simplify recruitment and augment control confounding factors between the two groups. The space had to be designed a minimum of three months before the study, had to be open to all Internet users, and updated at least weekly

Table 1. Sample description

\begin{tabular}{l|ll}
\hline & Chinese & British \\
\hline Age & Mean= 22 & Mean=25 \\
& Range 18-25 & Range 19-31 \\
Gender & $19 \mathrm{~F} ; 11 \mathrm{M}$ & $18 \mathrm{~F} ; 12 \mathrm{M}$ \\
Education Level & 18 Undergraduates & 6 Undergraduates \\
& 12 Postgraduates & 24 Postgraduates \\
Educational background & IT related: 18 Other: 12 & IT related: 2 Other: 28 \\
& & \\
\hline
\end{tabular}

Participants were identified with the MSN search engine. They were then contacted by e-mail to inform them of the study, verify that they matched the sampling requirements, and invite them to keep their space open to the researcher for the duration of the study.

\subsection{Results - Design patterns}

Design patterns were analysed by looking at differences in page layout, colour, functionality, and use of images.

\subsubsection{Page layout}

MSN spaces offered 6 standard layouts (Figure 3). We clustered them according to their degree of complexity (simple, medium and complex) by counting the number of structured spaces available to locate modules. Simple layouts included classical open grid pages, with a vertical top space for the title and an unstructured page underneath (i.e., layout 1). Layouts of medium complexity were composed of 3 spaces, a vertical title space and two bodies underneath which could be symmetrical (layout 2) or asymmetrical (layout 4 and 5). Complex layouts were 
composed of 4 spaces, a title space on the top and 3 body spaces which could be symmetrical (layout 3) or asymmetrical (layout 6).

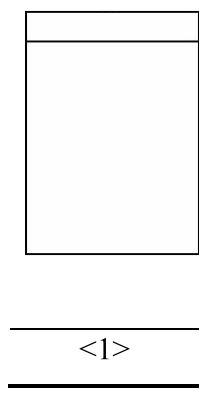

Fig. 3. Interface layouts

Table 2 reports the percentage of different layouts selected by Chinese and British students as a function of their complexity. British people preferred medium complexity layouts, whereas Chinese people preferred complex layouts. Layout 3, in particular, was selected by some $54 \%$ of Chinese participants but by only $12 \%$ of the British participants. The British preferred layout 4 (40\%), which was the second most preferred option for the Chinese (28\%).

Table 2. Percentages of layout selection

\begin{tabular}{c|cc}
\hline Layout complexity & UK & China \\
\hline Simple & $3 \%$ & $0 \%$ \\
Medium & $80 \%$ & $40 \%$ \\
Complex & $17 \%$ & $60 \%$ \\
\hline
\end{tabular}

Virtual spaces were further analysed by looking at modules placement within the place structure. This analysis suggested a generalised preference to place the profile window in the top-left corner of the page, right underneath the title bar, reserving the central part of the screen to blogs or photo-album according to individual preference for visual or verbal interaction styles. This tendency was stronger among Chinese users (87\%) than among British people (57\%), who were more inclined to experiment with different configurations.

\subsubsection{Colour}

The large spectrum of colours available to personalize virtual spaces was collapsed into two broad categories according to their psychological meaning: warm colours (part of the spectrum from red to orange and yellow) and cool colours (part of the spectrum from green to blue and purple). Research in visual perception suggests that colours engender different emotional reactions (Valdez and Mehrabian 1994). Warm colours are more activating, as they stimulates brain wave activity and skin conductance, while cool colours tend to be more deactivating or calming. The warm-cool association was found to be reliable among British and Chinese people (Byung-Kwan Lee et al. 2004).

The large majority of the spaces (75\%) displayed cool colours. Only $8 \%$ of them displayed warm colours and $16 \%$ used a combination of cool and warm colours. No differences emerged between British and Chinese spaces. 


\subsubsection{Functionality}

The types of modules available in the virtual space were counted. Blogs and photo-album were pervasive and appeared in all but 2 spaces. Profile was also a very common module selected by 29 Chinese and 27 British authors. Windows media player was present in some $65 \%$ of the spaces and the music list in $20 \%$. The author's cultural background did not influence the type of standard modules displayed in the spaces. However, Chinese students were more likely to create personalised modules than the British ones ( $83 \%$ versus $16 \%)$. Some $80 \%$ of the Chinese authors personalised the custom list into a friend list, but only $12 \%$ of the British authors did it. Cultural biases emerged also in the nature of other personalised functionalities. British students displayed lists of items reflecting their unique preferences, such as favourite movies, books, and drink lists. Chinese students preferred lists displaying famous quotes by other people, or provided less personalised information, such as click counters or weather forecast.

The analysis of the level of interactivity set in the space (e.g., whether the authors allowed readers to post comments on blogs and photo albums or not) did not reveal cultural differences. Overall, all of the British and $80 \%$ of the Chinese provided this facility, suggesting that communication is a fundamental driver of virtual spaces. In a follow up interview a Chinese participants who posted her poems on her space collecting over 100 responses commented "My poems not only brought more responses from my audience, but also helped me to build confidence".

\subsubsection{Images}

Chinese and English students used non verbal communication differently. The number of pictures in the main page of virtual spaces authored by Chinese students outnumbered those displayed by the British by a ratio of $4: 1$. Chinese students tended to use smaller images, including both icons and photos. British students favoured photos. The content of the photographs was also different. Chinese people preferred landscapes and group of people, whereas the British students tended to display mainly self-portrait.

The same number of Chinese and British students (28/30) displayed a picture in the profile section. However, for the Chineses some $70 \%$ of these images depicted a real person and the remaining ones depicted a cartoon character; whereas, in the British sample there were much more photos of real persons $(93 \%)$.

\subsection{Communication features}

Communication was analysed in terms of content, style, and self-disclosure.

\subsubsection{Content}

The 5 most recent entries of each blog were clustered into the taxonomy proposed by (Herring et al. 2004), as personal journals (on-line diaries in which the authors report on their lives, emotions and feelings), filters (commentaries on other people's web-sites) and knowledge blogs (notes on specific subject-matter). Contrary to the procedure followed in (Herring et al. 2004), we did not use a mixed category (indicating that the same entry belonged to different classes), but individual entries were clustered in more than one category if necessary. Results 
revealed that, with the exception of 1 Chinese student, all blog entries could be classified as on-line diaries. Filter blogs were common among Chinese ( $82 \%$ of the sample) but not among British students (18\%). Knowledge blogs were used only by the Chinese participants (15\%).

\subsubsection{Style}

Most of the Chinese blogs (26 out of 30) were written in Chinese and English. Two blogs were written only in Chinese and 2 in Japanese. All but one British blog were only in English, the exception being a blog which contained also some Spanish text. A qualitative analysis of blogs entry indicated that british students used a more informal communication style, characterised by the use of colloquial words, swearwords, and slang. On the contrary, swearing was very rare among Chinese students. It was also noted that British students tended to express their emotions more directly than Chinese students did.

\subsubsection{Self-disclosure}

Self-disclosure was measured by the number of items disclosed in profile. Signing up for profile, MSN users were prompted for a number of information which we clustered into 5 categories adapting the taxonomy proposed by (Lampe et al. 2007) for the analysis of self-disclosure in Facebook.

- Physical variables (photo, gender, age).

- Referents index (location, hometown, place lived, occupation, company name)

- Contact index (home address, home phone, mobile phone, e-mail).

- Interests index (interests, more about me, humour, favourite quote, fashion, music).

- Social (marital status, pets, date of birth, anniversary).

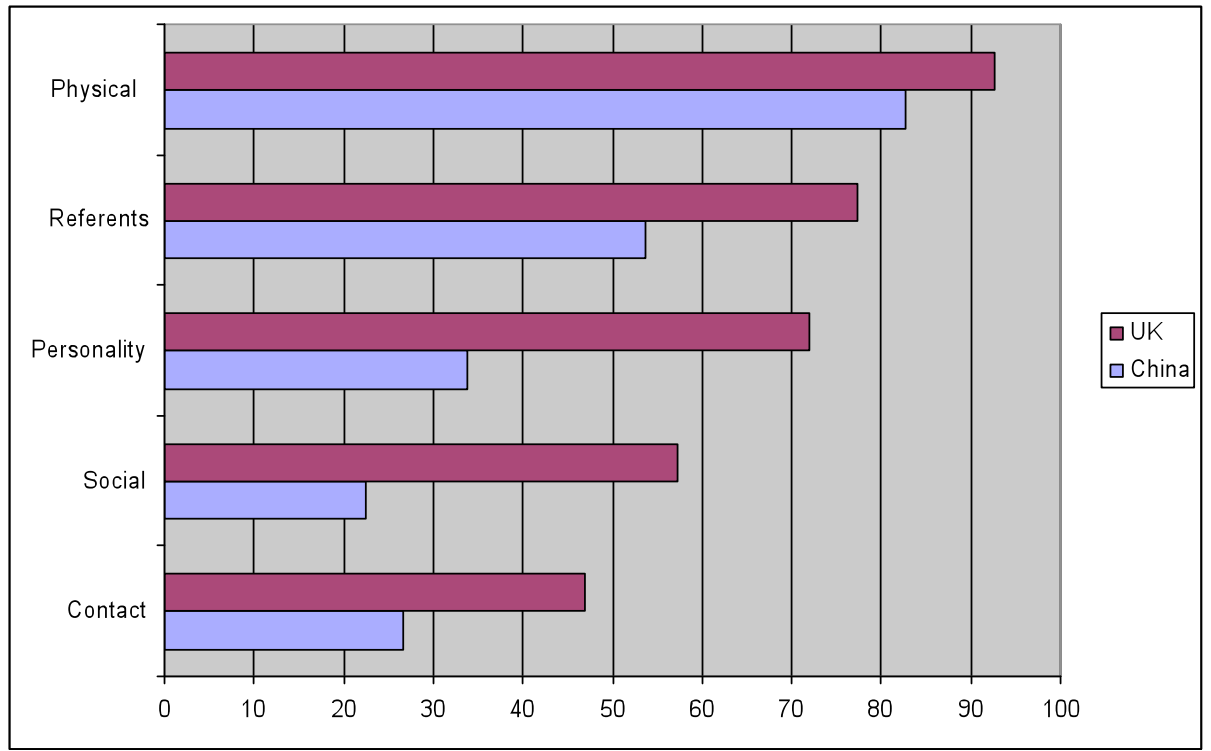

Fig. 4. Percentage of self-disclosure

The percentage of information disclosed in each dimension is illustrated in Figure

4. There is an evident difference in the quantity of information disclosed by 
British and Chinese in all dimensions. British students systematically disclosed more information than the Chinese students. The largest differences are in the disclosure of personality, social and contact information.

\subsection{Discussion}

The analysis provided support to the claim that cultural variations are manifested in virtual spaces (Hypothesis 1-b). The design of British and Chinese virtual spaces differed in systematic ways, and some of these differences closely mirrored cultural differences in real life or could be explained by instrumental characteristics of the different languages used for communication. Results are preliminary and the study has several limitations, including the small dimension of the sample and the convenience procedure used to recruit participants. Furthermore, the Chinese sample may not be considered fully representative as these people had been exposed to the western culture during their University studies. Despite these limitations, the reliability of the findings is supported by their consistency with theories of cultural studies (Hall 1976; Hofstede 2001; Marcus et al. 2006; Triandis 1989) and similarities with previous larger-scale studies of computer-mediated communication (Herring et al. 2004; Lampe et al. 2007).

As regards design at large, it was noted that Chinese people were characterised by less within-group variation in design than the British students. This observation is coherent with the principles of the inter-dependent self who value tradition and compliance (Markus and Kitayama 1991). A higher variability in design was noted in the British spaces, consistently with the principles of the independent self who is more open to individual differences and strives to affirm a unique identity. Furthermore, design and communication style of the Chinese spaces suggested that the author's may have put more effort in the design. Their spaces were more controlled and elaborated that the British ones. This difference can be explained by the nature of the inter-dependent self, who is more concerned about the evaluation of their peers, and as such may have put more effort in selfpresentation.

As regards specific design features, British and Chinese people tended to favour different layouts. British students were more likely to select a reverse 7 configuration (layout 4, Figure 4), which is a standard in web design. Chinese people preferred a symmetrical design (layout 3). There is an obvious functional reason for this shape, as vertical columns are best suited to accommodate logographic rather than orthographic writing (Dong and Salvendy 1999). However, we suggest that this shape may also have been favoured due to cultural familiarity. Layout 3 resembles indeed the typical New Year greeting couplets which Chinese hang on their house doors for good luck at the beginning of the Chinese year. Symmetry and balance are important for Chinese aesthetics, as can be seen, for instance, in their architecture, arts and philosophy. An association between symmetry and positive meaning in ideographs has been suggested (Takaki 2001).

Chinese people tended to design new functions more often than the British. This difference may be due to the different background of the sample, as more Chinese $(60 \%)$ than British $(6 \%)$ participants were students of IT related subjects. However, it can also reflect specific requirements of the Chinese sample which 
were not fulfilled by standard modules. We speculate that the modules offered by MSN spaces at the time of the study were biased towards western users.

Consequently, Chinese had to create specific functions to fulfil their own needs. A typical example of a cultural need was the Friend list, which appeared more frequently in Chinese spaces than in British ones (this module is now offered by default by most SNP's spaces). A friend list can be more important for persons with an inter-dependent self as it provides a direct insight into the social network of the author. The fact that people spend energy and time in building new functions can also be explained by the increased commitment in self-presentation, previously discussed.

It is also worth noting that at the time of the study there was a much larger availability of peer-to-peer resources for creating new MSN modules in Chinese, rather than in English. A Google search with the English phrase 'MSN spaces tutorial' returned most documents from official Microsoft web-sites explaining how to use existing features, rather than how to build new ones. On the contrary, the same search in Chinese retrieved several documents from personal blogs and web-pages reporting simple tips for creating 'amazing and special' MSN spaces, as quoted in one of the available sources.

The trend evinced in the analysis of the use of pictures matched Hall's proposition that cultures vary according to the contextual knowledge a person should possess for successful communication (Hall 1976). In Chinese virtual spaces more importance was placed in non-verbal aspects of communication and their spaces displayed more images than the British ones. The difference between low-context and high-context communication was also evident in verbal communication. Chinese people were more polite, and authored filter blogs more often than the British. The frequent use of filter blogs reflects the nature of the inter-dependent self, which gains meaning through associations and relationships rather than direct self-expression (Markus and Kitayama 1991).

Cultural differences also emerged in the quantity and quality of the information disclosed in the profile. British tended to disclose more individuating information, consistent with the nature of the independent self. The reduced number of personal items found in the Chinese profiles can be regarded as a deliberate decision not to disclose as people in eastern countries tend not to disclose personal information to strangers (Markus and Kitayama 1991). On the other hand, the difference in self-disclosure can be regarded as a consequence of design ethnocentrism. Indeed, it could be argued that most of the items required in the profile were typical dimensions of an independent self. Thus, they may have been perceived as irrelevant, or even inappropriate, for the Chinese. A prototypical example of this mismatch is the item pets. It was filled in by some $75 \%$ of British students, but only by $20 \%$ of the Chinese, reflecting different attitudes towards domestic animals in the two countries.

\section{Survey study}

A survey study was designed to investigate some of the issues emerged in Study 1 , suggesting a difference in effort devoted to the design of virtual spaces, communication style and likelihood of self-disclosure between British and Chinese students. The survey expanded the contraposition between hypothesis 1-a 
and hypothesis $1-b$ (attributing variable importance to culture) by contrasting the effect of personality with that of culture. For each dimension (effort, communication style, and self-disclosure) we generated two hypotheses predicting cultural and personality effects (Table 3). Cultural hypotheses were based on the theoretical background discussed so far in the paper and on results of study 1 . Personality hypothesis were based on the Big Five Model, a framework of personality dimensions considered to be consistent among cultures (John and Srivastava 1999). This five-factor model encompasses dimensions of extraversion (e.g., talkative and energetic), agreeableness (e.g., kind and altruist), conscientiousness (organized and competent), neuroticism (moody and anxious), and openness to experience (fantasy and having wide interests).

Table 3. Cultural and personality hypotheses

\begin{tabular}{|l|l|}
\hline \multirow{5}{*}{ Effort } & $\begin{array}{l}\text { Cultural hypothesis (HE1): } \\
\text { Chinese people will put more effort in the design of their } \\
\text { space, as they tend to be more concerned about the results of } \\
\text { their actions on members of their in-group. }\end{array}$ \\
\cline { 2 - 2 } $\begin{array}{l}\text { Personality hypothesis (HE2): } \\
\text { A correlation between commitment and conscientiousness is } \\
\text { expected because this dimension is associated with discipline, } \\
\text { need for achievement, and deliberation. }\end{array}$ \\
\hline Style & $\begin{array}{l}\text { Cultural hypothesis (HCS1): } \\
\text { Chinese people will be more polite and more formal in their } \\
\text { writing as this reflects their face-to-face communication style }\end{array}$ \\
\cline { 2 - 3 } Self-disclosure & $\begin{array}{l}\text { Personality hypothesis (HCS2): } \\
\text { A correlation between politeness and agreeableness is } \\
\text { expected. }\end{array}$ \\
\hline $\begin{array}{l}\text { Cultural hypothesis (HSD1): } \\
\text { British people will disclose more individuating information } \\
\text { (who I am), whereas Chinese people will disclose more social } \\
\text { information (who my friends are). }\end{array}$ \\
\cline { 2 - 2 } $\begin{array}{l}\text { Personality hypothesis (HSD2): } \\
\text { A correlation between quantity of disclosed information and } \\
\text { extraversion is expected. }\end{array}$ \\
\hline
\end{tabular}

\subsection{Method}

Data were collected by an on-line questionnaire, advertised by e-mail to the students of the University of Manchester. The questionnaire was kept on line for 3 weeks in August 2006. All users of MSN spaces, independently of their ethnical origin, were invited to participate. In this paper, however, we report only on respondents from the UK and China.

Personality was measured by the Big Five Inventory (John and Srivastava 1999). People's behaviour and opinions were collected by a questionnaire developed and pilot tested for the purpose of this research. It included 4 sections: personal effort, design features and communication style, and personal data. 


\subsection{Results}

Complete responses were obtained from 274 people. A total of 159 respondents matched the sampling requirements and were used for the analysis ( 83 were from the UK and 76 from China). Some $60 \%$ of the participants were women. Most of them (54\%) were aged between 18 and 21 years old, 36\% between 22 and 24 years and $10 \%$ were over 25 years old. Half of the sample had their space for more than one year (51\%), 27\% had it for a period of time between 6 months and 1 year, $10 \%$ for 3-6 months, and $12 \%$ for less than three months. Some $40 \%$ of the sample pursued an IT related degree. No statistical differences were found between the two cultures as regards length of experience with MSN spaces and educational background (IT related vs. other).

\subsubsection{Effort}

The cultural hypothesis (HE1, Table 3) was tested by comparing self-reports on how often students updated their web sites, how long for, and if they had created new modules, between the two nationality groups. The first two items were analysed with Mann-Whitney $U$ tests which returned significant results. Chinese people tended to update their space more often $(\mathrm{U}=1263.5, \mathrm{~N}=159, p<.001)$ and engaged in this activity longer than the British people did $(\mathrm{U}=1481.5, \mathrm{~N}=$ $158, p<.01)$. Furthermore, some $48 \%$ of Chinese declared having created new modules, whereas only $14 \%$ of the British did so. The difference is significant $\left(\chi^{2}\right.$ $=18.86, p<.001)$.

The personality hypothesis (HE2) was tested by correlating the items assessing frequency of update and time spent updating the space with mean scores to the personality dimension of consciousness. No significant effects were returned (Spearman rho $p=\mathrm{n} . \mathrm{s}$ ), suggesting that consciousness was independent of the effort devoted to the design of virtual spaces. Furthermore, no personality differences on the dimension of consciousness were found between people who created and people who did not created new modules.

\subsubsection{Communication style}

To test the relative effect of personality and culture on communication style, participants were invited to assess their blog on a semantic differential scale composed of 5 pairs of adjectives on a 7 point-scale (Table 4). The cultural hypothesis, HCS1, was tested by entering these adjectives as dependent variables into a Manova with culture (2) as the between subjects factors. The multivariate effect of culture was significant indicating that overall people from China perceived their communication style differently that people from the UK $\left(F_{(5,150)}=\right.$ $5,45, p<.001$ ). The analysis of the simple effects (Anovas) indicated that the major differences were in the evaluation of happiness and politeness. Specifically, Chinese people tended to perceive their blog as more polite and less happy than the British people did. Mean values and standard errors (in brackets) for each item are reported in Table 3, alongside the $p$ values returned from the univariate Anovas. The dimension of formality returned only a marginal effect which anyway was in the expected direction. 
Table 4. Perception of communication style in blog writing

\begin{tabular}{|l|l|l|l|}
\hline & Chinese & British & p \\
\hline formal-informal & $5.16(.23)$ & $5.75(.23)$ & $=.07$ \\
\hline sad-happy & $4.35(21)$ & $5.02(.20)$ & $<.05$ \\
\hline impolite-polite & $4.89(.22)$ & $3.89(.20)$ & $<.001$ \\
\hline boring-interesting & $5.00(.19)$ & $4.74(.18)$ & n.s. \\
\hline introvert-extrovert & $4.37(.20)$ & $4.71(.21)$ & n.s.. \\
\hline
\end{tabular}

To test the effect of personality (HCS2), all items addressing communication style were correlated with the mean scores to the personality factors. As expected, a significant correlation emerged between agreeableness and perceived level of politeness in communication style $(r=.30, p<.001)$. Other significant correlations were found. The evaluation of the blog style on the introversionextraversion dimensions positively correlated with personality traits $(r=.41, p<$ $.001)$. Self-appraisal of blog interest correlated with openness $(r=.31, p<.05)$, and perceived happiness style negatively correlated with neuroticism $(r=.-.26, p$ $<.05)$.

\subsubsection{Self-disclosure}

Self-discloure was operationalised as the number of information items disclosed in profile, following the procedure applied in Study 1. Results are illustrated in Figure 5.

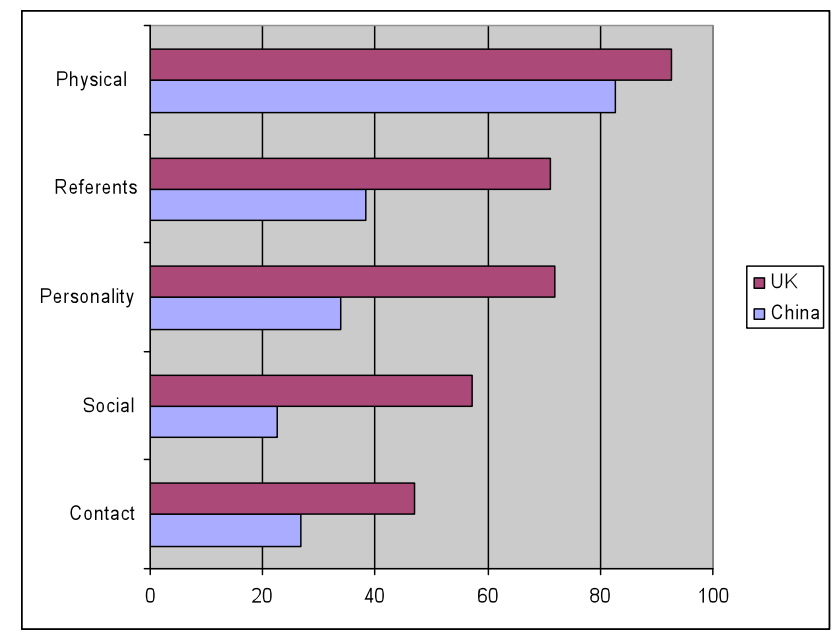

Fig. 5. Percentage of information disclosed

The cultural hypothesis (HSD1) was strongly supported. Non parametric tests evinced significant differences for all categories. British people were more likely to disclose information than the Chinese. Furthermore, the majority of Chinese people $(61 \%)$ declared to have a friend list on their web-site, whereas only $37 \%$ of the British did so $\left(\chi^{2}=7.16, p<.05\right)$.

The correlation between total item disclosed and the extraversion scores revealed only a marginal association $(r=16, p=.06)$, suggesting that the role of personality on self-disclosure in profile is weak (HSD2). 


\subsection{Discussion}

Table 5 reports a summary of the findings from the survey studies. The hypotheses which were supported are labelled with a plus, whereas rejected hypotheses are labelled with a minus. All of the cultural hypotheses were supported suggesting that the cultural background of the respondents had a relevant influence on on-line self-presentation. On the contrary, the role of personality was weaker. With the only exception of the relationship between personality and communication style, no other associations were found.

Table 5. Result summary

\begin{tabular}{|l|l|l|}
\hline & Culture & Personality \\
\hline Effort & + & - \\
\hline Communication Style & + & + \\
\hline Self-disclosure & + & - \\
\hline
\end{tabular}

The reliability and generality of the study is limited by the sample size and the sampling procedure, yet the trend of results is consistent with study 1 and the theoretical background previously discussed.

\section{Discussion}

As communication technology provides increasingly opportunities to reach out a potentially worldwide audience it becomes fundamental to understand on-line cross-cultural encounters and negotiation as well as implicit and explicit design associations and meanings. The studies reported in this paper have provided support to the hypothesis that the cultural identity of the user pervades on-line self-presentation, influencing communication style and design preferences. From this preliminary exploration it emerged that, in this context, culture could be even more important than the author's personality.

The participants in our studies were prototypical inhabitants of the global village: young and educated people. The Chinese sample was composed of people who studied in the UK and was proficient in at least two languages: yet, they were different from the British born students. We expect that these differences will be enhanced in people who have not been exposed to different cultural contexts, and in older people. Further research addressing different samples is necessary to address the reliability of cultural differences in virtual spaces design.

The studies reported in this paper suggested that the current design of SNP's may still be biased towards habits and needs of western users. Examples of such a design ethnocentrism are both functional (Chinese people were more likely to create ad-hoc applications than the British, as if the current offer did not fulfil their own requirements) and communicative (Chinese people tended to disclose less information in the profile fields than the British, as if that information was not relevant to them). The difference in quantity and quality of information disclosed in virtual spaces profile is more important that it first may appear. Recent research has indeed demonstrated a link between the quantity of personal information disclosed in profile and the number of friends a user will have listed in Facebook (Lampe et al. 2007). Grounding these findings in a comprehensive analysis and 
integration of signalling theory, common-ground theory, and transaction theory, the authors suggest that profile fields foster social connections.

It is a big challenge and a moral responsibility for the HCI community to find effective methods and techniques to address cultural differences of users in the design of technology. There is an urgent need to verify how much of the theoretical and methodological apparatus driving interaction design is universal and how much is biased towards specific cultural values and habits of western people. Our research is a preliminary step towards a theory and a practice of cultural-aware design which is necessary if we are to decrease the digital divide and offer each user, independently of their ethnical and cultural background, a comfortable place in the global village.

Designing for a global population requires understanding differences and similarities between heterogeneous groups of people. In this paper we propose a methodology to understand and model cultural differences in technology usage. Our proposal integrates theory-led research and exploratory empirical studies to collect evidence on cultural differences in computer-mediated communication. The theoretical framework builds on an integration of the theory of cultural selfconstrual (Markus and Kitayama 1991) and Hall's concept of context (1976). We propose that this background should be considered by HCI research, alongside the main theoretical framework applied so far based on Hofstede research. The paper also proposes a new target for cultural research, suggesting that virtual spaces may offer a suitable domain to identify cultural markers in interaction design.

\section{Acknowledgements}

I would like to thank Di Hong for her help with data collection. My gratitude also goes to Liping Zhao and Sri Kurniawan who have been instrumental in helping me making sense of the data reported in this paper.

\section{References}

Banaji M (1994). The Self in Social Contexts. Annual Review of Psychology 45(1):297-332

Barber W, Badre A (1998) Culturability: The merging of culture and usability. 4th Conference on Human Factors and The Web.

Blom J, Monk (2003) Theory of personalization of appearance: Why users personalize their pcs and mobile phones. Human-Computer Interaction 18(3):193-228.

Byung-Kwan Lee, Hong J-Y, Lee W-N. (2004) How Attitude Toward the Web Site Influences Consumer Brand Choice and Confidence While Shopping Online. Journal of Computer-Mediated Communication. 9(2).

De Angeli A, Athavankar U, Joshi A et al. (2004) Introducing ATMs in India: a contextual inquiry. Interacting with Computers, 16(1):29-44.

De Angeli A, Kyriakoullis L (2006) Globalisation vs. localisation in e-commerce: cultural-aware interaction design, 23-26 May; Venice. ACM. p 250-253.

Dearden AM, Dunckley L, Best, M et al (2007) Socially Responsible Design in the Context of International Development. INTERACT (2) 2007: 694-695

Dong J, Salvendy G. (1999) Designing menus for the Chinese population: horizontal or vertical? Behaviour \& Information Technology 18(6):467471 . 
Efendioglu AM, Yip VF (20040 Chinese culture and e-commerce: an exploratory study. Interacting with Computers 16(1):45-62.

Goffman E (1959) The Presentation of Self in Everyday Life. New York: Doubleday/Anchor books.

Gutchess AH, Welsh RC, Boduroğlu A (2006) Cultural differences in neural function associated with object processing. Cognitive, affective and bahavioural neuroscience 6(2):102-109.

Hall ET (1976) Beyond Culture. New York: Doubleday.

Herring S, Scheidt L, Bonus S et al. (2004) Bridging the gap: A genre Analysis of weblogs; 2004; Big Island, Hawaii. IEEE.

Hofstede G. (2001) Culture's consequences. California: Sage, Thousand Oaks.

John OP, Srivastava S (1999) The Big-Five Trait Taxonomy: History, Measurement, and Theoretical Perspectives. In: Pervin L, John OP, editors. Handbook of personality: Theory and research (2nd ed). New York: Guilford. p 102-138.

Lampe C, Ellison N, Steinfield C (2007) A familiar Face(book): Profile elements as signals in an on-line network; San Jose, CA, USA. ACM. p 435-444.

Lea M (1991) Computer-mediated communication, de-individuation and group decision-making. International journal of man-machine studies 34(2):283.

Leary MR (1996) Self-presentation : impression management and interpersonal behavior. Boulder, CO: Westview Press.

Marcus A (2002) User-interface design, culture and the future. In: De Marsico M, Levialdi S, E. P, editors; 2002; Trento, Italy. ACM press. p 15-27.

Marcus B, Franz M, Schütz A (2006) Personality in cyberspace: personal Web sites as media for personality expressions and impressions. Journal of personality and social psychology 90(6):1014-1031.

Markus HR, Kitayama S (1991) Culture and the Self: Implications for Cognition, Emotion, and Motivation. Psychological Review 98(2):224-253.

Nisbett RE (2003) The geography of thought: How asians and westeners think differently.... and why. New York: Free Press.

Postmes T, Spears R, Lea M (1998) Breaching or building social boundaries? SIDE-effects of computer-mediated communication. Communication Research 26(6):689-715.

Smith A, Yetim F (2004) Global human-computer systems: cultural determinants of usability. Interacting with Computers 16(1):1-5.

Sproull L, Kiesler S (1992) Connections: New Ways of Working in the Networked Organization: MIT Press.

Tajfel H, Turner JC (1986) The social identity theory of inter-group behavior. In: Worchel S, Austin LW, editors. Psychology of Intergroup Relations. Chigago: Nelson-Hal. p 7-24.

Takaki R (2001) Towards a Reformation of Chinese Ideographs. Forma 16:289305.

Triandis H (1989) The self and social behavior in differing cultural contexts. Psychological review 96(3):506.

Turner JC (1987) Rediscovering the social group: A self-categorization theory. Oxford UK: Basil Blackwell.

Valdez P, Mehrabian A (1994) Effects of Color on Emotions. Journal of Experimental Psychology: General.

Wood AF, Smith MJ (2005) On-line communication - 2nd Edition. Mahwah, NJ: Lawrence Erlbaum Associates. 
